\title{
On the well-posedness problem of the electrorheological fluid equations
}

Huashui Zhan ${ }^{1 *}$

\section{"Correspondence:} huashuizhan@163.com

'School of Applied Mathematics, Xiamen University of Technology, Xiamen, P.R. China

\section{Springer}

\begin{abstract}
A kind of electrorheological fluid equations, with the orientated convection terms

$$
u_{t}=\operatorname{div}\left(a(x)|\nabla u|^{p(x)-2} \nabla u\right)+\vec{b}(x) \cdot \nabla u^{q}, \quad(x, t) \in \Omega \times(0, T),
$$

is considered. It is supposed that $a(x)>0$ when $x \in \Omega, a(x)=0$ when $x \in \partial \Omega$. If there is interaction between the diffusion coefficient $a(x)$ and the convective coefficient $\vec{b}$, the stability of the solutions can be proved without any boundary condition.
\end{abstract}

MSC: $35 \mathrm{~K} 65 ; 35 \mathrm{~K} 55 ; 35 \mathrm{Q} 35$

Keywords: The electrorheological fluid equation; Orientated convection term; The well-posedness problem; Boundary value condition

\section{Introduction and the main results}

Consider the diffusion equation with an orientated convection term

$$
u_{t}=\operatorname{div}\left(a(x)|\nabla u|^{p(x)-2} \nabla u\right)+\vec{b}(x) \cdot \nabla u^{q}, \quad(x, t) \in Q_{T}=\Omega \times(0, T) .
$$

with the initial value

$$
u(x, 0)=u_{0}(x), \quad x \in \Omega,
$$

and the boundary value

$$
u(x, t)=0, \quad(x, t) \in \partial \Omega \times(0, T) .
$$

Here $1<p(x) \in C(\bar{\Omega}), q \geq 1, a(x) \in C^{1}(\bar{\Omega}), \vec{b}=\left\{b^{i}\right\}, b^{i}(x) \in C^{1}(\bar{\Omega}), \Omega \subset \mathbb{R}^{N}$ is a bounded domain with a smooth boundary $\partial \Omega$. Equation (1.1) arises in electrorheological fluids theory [1]. If $\vec{b}(x)=0, a(x)=1$ for all $x \in \bar{\Omega}$, the existence and uniqueness results of Eq. (1.1) have been widely researched, one may refer to [2-7] and the references therein. If $p(x)=p$, the equations are known as non-Newtonian fluid equations, and have been studied by many mathematicians, one may refer to [8] and the references therein. All these papers show that the uniqueness and the stability of the solutions can be proved if the Dirichlet boundary value condition (1.3) is imposed. In this paper, we will show that, if the diffusion coefficient $a(x)$ is degenerate on the boundary and, moreover, there is interaction between the

(c) The Author(s) 2018. This article is distributed under the terms of the Creative Commons Attribution 4.0 International License (http://creativecommons.org/licenses/by/4.0/), which permits unrestricted use, distribution, and reproduction in any medium, provided you give appropriate credit to the original author(s) and the source, provide a link to the Creative Commons license, and indicate if changes were made. 
diffusion process and the convection process, then the stability of the solutions may be established without any boundary value condition.

We denote

$$
p_{+}=\max _{x \in \bar{\Omega}} p(x), \quad p_{-}=\min _{x \in \bar{\Omega}} p(x)
$$

we assume that $p_{-}>1, a(x)>0$ in $\Omega$,

$$
a(x)=0, \quad x \in \partial \Omega
$$

By this token, if $\vec{b}(x)=0$, our previous papers [9-11] showed that the boundary value condition (1.3) may be redundant, the uniqueness of the weak solutions can be proved only depending on the initial value (1.2). Accordingly, in this paper, we will construct a suitable test function to obtain the stability of the weak solutions independent of the boundary value condition (1.3). We give the basic concepts and the main results now.

Lemma 1.1 If $q_{1}(x)$ and $q_{2}(x)$ are real functions with $\frac{1}{q_{1}(x)}+\frac{1}{q_{2}(x)}=1$ and $q_{1}(x)>1$, then, for any $u \in L^{q_{1}(x)}(\Omega)$ and $v \in L^{q_{2}(x)}(\Omega)$,

$$
\left|\int_{\Omega} u v d x\right| \leq 2\|u\|_{L^{q_{1}(x)}(\Omega)}\|v\|_{L^{q_{2}(x)}(\Omega)} .
$$

\section{Lemma 1.2}

$$
\begin{aligned}
& \text { If }\|u\|_{L^{p(x)}(\Omega)}>1 \text {, then }\|u\|_{L^{p(x)}(\Omega)}^{p_{-}} \leq \int_{\Omega}|u|^{p(x)} d x \leq\|u\|_{L^{p(x)}(\Omega)}^{p_{+}} . \\
& \text {If }\|u\|_{L^{p(x)}(\Omega)}<1 \text {, then }\|u\|_{L^{p(x)}(\Omega)}^{p_{+}} \leq \int_{\Omega}|u|^{p(x)} d x \leq\|u\|_{L^{p(x)}(\Omega)}^{p_{-}} .
\end{aligned}
$$

Lemma 1.1 and Lemma 1.2 can be found in [12].

Definition 1.3 If a nonnegative function $u(x, t)$ satisfies

$$
\begin{aligned}
& u \in L^{\infty}\left(Q_{T}\right), \quad u_{t} \in \in L^{p_{+}^{\prime}}\left(0, T ; W^{-1, p_{+}{ }^{\prime}}(\Omega)\right), \\
& a(x)|\nabla u|^{p(x)} \in L^{\infty}\left(0, T ; L^{1}(\Omega)\right),
\end{aligned}
$$

and, for any function $g(s) \in C^{1}(\mathbb{R}), g(0)=0, \varphi_{1} \in C_{0}^{1}(\Omega), \varphi_{2} \in L^{\infty}\left(0, T ; W_{\mathrm{loc}}^{1, p(x)}(\Omega)\right)$,

$$
\begin{gathered}
\iint_{Q_{T}}\left[\frac{\partial u}{\partial t} g\left(\varphi_{1} \varphi_{2}\right)+a(x)|\nabla u|^{p(x)-2} \nabla u \cdot \nabla g\left(\varphi_{1} \varphi_{2}\right)\right. \\
\left.+u^{q}\left(b_{x_{i}}^{i} g\left(\varphi_{1} \varphi_{2}\right)+b^{i}(x) g g_{x_{i}}\left(\varphi_{1} \varphi_{2}\right)\right)\right] d x d t=0,
\end{gathered}
$$

then we say $u(x, t)$ a solution of Eq. (1.1) with the initial value (1.2), and the initial value (1.2) is satisfied in the sense

$$
\lim _{t \rightarrow 0} \int_{\Omega} u(x, t) \phi(x) d x=\int_{\Omega} u_{0}(x) \phi(x) d x=0,
$$

for any $\phi(x) \in C_{0}^{\infty}(\Omega)$. 
Here, $p_{+}^{\prime}=\frac{p_{+}}{p_{+}-1}, b_{x_{i}}^{i}=\frac{\partial b^{i}(x)}{\partial x_{i}}, g_{x_{i}}=\frac{\partial g}{\partial x_{i}}$ as usual. In this paper, the existence of the nonnegative solution is proved firstly.

Theorem 1.4 If $p_{-}>1,1 \leq q<p_{+}$,

$$
0 \leq u_{0} \in L^{\infty}(\Omega), \quad a(x)\left|\nabla u_{0}\right|^{p(x)} \in L^{1}(\Omega), \quad i=1,2, \ldots, N
$$

then Eq. (1.1) with initial value (1.2) has a nonnegative weak solution $u$.

In particular, when $a(x)=d^{\alpha}(x), d(x)=\operatorname{dist}(x, \partial \Omega)$ is the distance function from the boundary, $0<\alpha<p^{-}-1$ is a constant, similar to the proof of Theorem 1.1 in [13], we can show that there is a constant $\gamma \geq 1$ such that the weak solution $u$ in Theorem 1.4 satisfies

$$
\int_{Q_{T}}|\nabla u|^{\gamma} d x d t \leq c
$$

Similarly, if we impose some restrictions on $a$, we also can show (1.9) is true, then the boundary value condition (1.3) is valid in the sense of the trace. However, in general, we cannot define the trace of $u$ on the boundary. Accordingly, instead of being interested in the boundary value condition, we would pay a close attention on the stability of the weak solutions without any boundary value condition. By the innovative definition of the weak solution above, we can prove the following main results.

Theorem 1.5 If $q \geq 1, u$ and $v$ are two nonnegative weak solutions of Eq. (1.1), and

$$
\begin{aligned}
& \int_{\Omega}\left|\frac{b^{i}(x) \nabla a(x)}{a(x)}\right|^{2} d x<\infty, \quad\left|b_{i}(x) a^{-\frac{1}{p(x)}}(x)\right| \leq c, \\
& \int_{\Omega} a^{-(p(x)-1)}(x) d x<\infty
\end{aligned}
$$

then

$$
\int_{\Omega}|u(x, t)-v(x, t)| d x \leq c \int_{\Omega}\left|u_{0}(x)-v_{0}(x)\right| d x, \quad \text { a.e. } t \in[0, T) .
$$

Theorem 1.6 If $q \geq 1, u$ and $v$ are two nonnegative weak solutions of Eq. (1.1),

$$
\int_{\Omega} a^{-\frac{1}{p(x)-1}}(x) d x<\infty
$$

and

$$
\frac{c}{\lambda}\left(\lambda \int_{\Omega \backslash \Omega_{\lambda}}|\nabla a|^{p(x)} d x\right)^{\frac{1}{p_{+}}} \leq c,
$$

then the stability of the weak solutions is true in the sense of (1.12). Here $\Omega_{\lambda}=\{x \in \Omega$ : $a(x)>\lambda\}$.

Remark 1.7 If $1<p_{-} \leq p(x) \leq p_{+} \leq 2$, then the condition (1.14) is satisfied. 
Theorem 1.8 If $q \geq 1, a(x)$ satisfies (1.13), and for small enough $\lambda>0, u(x, t)$ and $v(x, t)$ satisfy

$$
\frac{1}{\lambda}\left(\int_{\Omega \backslash \Omega_{\lambda}} a(x)|\nabla u|^{p(x)} d x\right)^{\frac{1}{q_{+}}} \leq c, \quad \frac{1}{\lambda}\left(\int_{\Omega \backslash \Omega_{\lambda}} a(x)|\nabla \nu|^{p(x)} d x\right)^{\frac{1}{q_{+}}} \leq c,
$$

then the stability (1.12) is true. Here $q(x)=\frac{p(x)}{p(x)-1}, q_{+}=\max _{x \in \bar{\Omega}} q(x)$.

In a word, the innovation of the definition of the weak solutions is that we use the test function with the form $\varphi_{1} \varphi_{2}$ instead of the usual one $\varphi$ to overcome the difficulty that the weak solutions may lack the regularity to define the trace. The weak solutions the existence of the weak solutions is proved by the usual parabolically regularized method. The stability of the weak solutions are proved by choosing some special test functions.

\section{The weak solutions dependent on the initial value}

Consider the following approximate problem:

$$
\begin{aligned}
& u_{\varepsilon t}-\varepsilon \operatorname{div}\left(\left|\nabla u_{\varepsilon}\right|^{p_{+}-2} \nabla u_{\varepsilon}\right)-\operatorname{div}\left(a(x)\left|\nabla u_{\varepsilon}\right|^{p(x)-2} \nabla u_{\varepsilon}\right)-\vec{b}(x) \cdot \nabla u_{\varepsilon}^{q} \\
& \quad=0, \quad(x, t) \in Q_{T}, \\
& u_{\varepsilon}(x, t)=0, \quad(x, t) \in \partial \Omega \times(0, T), \\
& u_{\varepsilon}(x, 0)=u_{\varepsilon, 0}(x), \quad x \in \Omega,
\end{aligned}
$$

where $0 \leq u_{\varepsilon, 0} \in C_{0}^{\infty}(\Omega),\left|u_{\varepsilon, 0}\right|_{L^{\infty}(\Omega)} \leq\left|u_{0}\right|_{L^{\infty}(\Omega)}, a(x)\left|\nabla u_{\varepsilon, 0}\right|^{p_{+}}$uniformly is convergent to $a(x)\left|\nabla u_{0}(x)\right|^{p_{+}}$in $L^{1}(\Omega)$. Then there is an unique nonnegative solution $u_{\varepsilon} \in L^{p_{+}}(0, T$; $\left.W_{0}^{1, p_{+}}(\Omega)\right)[6]$.

By the maximum principle ([8], p. 150), we have

$$
\left\|u_{\varepsilon}\right\|_{L^{\infty}\left(Q_{T}\right)} \leq c
$$

Since

$$
\begin{aligned}
\iint_{Q_{T}}\left|u_{\varepsilon} \vec{b}(x) \cdot \nabla u_{\varepsilon}^{q}\right| d x d t & =q \iint_{Q_{T}}\left|u_{\varepsilon}^{q} \vec{b}(x) \cdot \nabla u_{\varepsilon}\right| \\
& \leq \frac{\varepsilon}{2} \iint_{Q_{T}}\left|\nabla u_{\varepsilon}\right|^{p_{+}} d x d t+c(\varepsilon),
\end{aligned}
$$

by multiplying (2.1) with $u_{\varepsilon}$ and integrating it over $Q_{T}$, we easily obtain

$$
\frac{1}{2} \int_{\Omega} u_{\varepsilon}^{2} d x+\varepsilon \iint_{Q_{T}}\left|\nabla u_{\varepsilon}\right|^{p^{+}} d x d t+\iint_{Q_{T}} a(x)\left|\nabla u_{\varepsilon}\right|^{p(x)} d x d t \leq c .
$$

For any $\Omega_{\lambda} \subset \subset \Omega$, since $p_{-}=\min _{x \in \bar{\Omega}} p(x)>1$, by (2.5),

$$
\int_{0}^{T} \int_{\Omega_{\lambda}}\left|\nabla u_{\varepsilon}\right| d x d t \leq c\left(\int_{0}^{T} \int_{\Omega_{\lambda}}\left|\nabla u_{\varepsilon}\right|^{p_{-}} d x d t\right)^{\frac{1}{p-}} \leq c(\lambda)
$$


and

$$
\varepsilon \iint_{Q_{T}}\left|\nabla u_{\varepsilon}\right|^{p_{+}} d x d t \leq c .
$$

For any $v \in L^{p_{+}}\left(0, T ; W_{0}^{1, p_{+}}(\Omega)\right),\|v\|_{L^{p_{+}}\left(0, T ; W_{0}^{1, p_{+}}(\Omega)\right)}=1$. We have

$$
\begin{gathered}
\left\langle u_{\varepsilon}, v\right\rangle+\varepsilon \iint_{Q_{T}}\left|\nabla u_{\varepsilon}\right|^{p_{+}-2} \nabla u_{\varepsilon} \nabla v d x d t \\
+\iint_{Q_{T}} a(x)\left|\nabla v_{\varepsilon}\right|^{p(x)-2} \nabla u_{\varepsilon} \nabla v d x d t \\
\quad+\iint_{Q_{T}} u^{q}\left(b_{x_{i}}^{i}(x) v+b^{i}(x) v_{x_{i}}\right) d x d t .
\end{gathered}
$$

This formula makes sense because $u_{\varepsilon} \in L^{p_{+}}\left(0, T ; W_{0}^{1, p_{+}}(\Omega)\right) \cap L^{\infty}\left(Q_{T}\right)$.

By (2.8), using the Young inequality, we can show that

$$
\begin{aligned}
\left|\left\langle u_{\varepsilon}, v\right\rangle\right| \leq c & {\left[\varepsilon \iint_{Q_{T}}\left|\nabla u_{\varepsilon}\right|^{p_{+}} d x d t+\iint_{Q_{T}} a(x)\left|\nabla u_{\varepsilon}\right|^{p(x)} d x d t\right.} \\
& \left.+\iint_{Q_{T}}\left(|v|^{p_{+}}+|\nabla v|^{p_{+}}\right) d x d t+1\right] \leq c,
\end{aligned}
$$

then

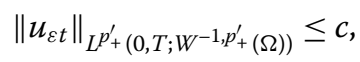

where $p_{+}^{\prime}=\frac{p_{+}}{p_{+}-1}$.

Now, for any $\varphi \in C_{0}^{1}(\Omega), 0 \leq \varphi \leq 1$, for any $v \in L^{p_{+}}\left(0, T ; W_{0}^{1, p_{+}}(\Omega)\right)$,

$$
\begin{aligned}
\left\langle\left(\varphi u_{\varepsilon}\right)_{t}, v\right\rangle= & \left\langle\varphi u_{\varepsilon t}, v\right\rangle=\left\langle u_{\varepsilon t}, \varphi v\right\rangle \\
= & \varepsilon \iint_{Q_{T}}\left|\nabla u_{\varepsilon}\right|^{p_{+}-2} \nabla u_{\varepsilon} \nabla(\varphi v) d x d t \\
& +\iint_{Q_{T}} a(x)\left|\nabla u_{\varepsilon}\right|^{p(x)-2} \nabla u_{\varepsilon} \nabla(\varphi v) \\
& +\iint_{Q_{T}} u^{q}\left(b_{x_{i}}^{i}(x) v \varphi+b^{i}(x)(v \varphi)_{x_{i}}\right) d x d t .
\end{aligned}
$$

Similarly, we can show that

$$
\begin{aligned}
\left|\left\langle\left(\varphi u_{\varepsilon}\right)_{t}, v\right\rangle\right|= & \left\langle u_{\varepsilon t}, \varphi v\right\rangle \\
\leq & c\left[\varepsilon \iint_{Q_{T}}\left|\nabla u_{\varepsilon}\right|^{p_{+}} d x d t+\iint_{Q_{T}} a(x)\left|\nabla u_{\varepsilon}\right|^{p(x)} d x d t\right. \\
& \left.+\iint_{Q_{T}}\left(|v|^{p_{+}}+|\nabla v|^{p_{+}}\right) d x d t+1\right] \leq c,
\end{aligned}
$$

then

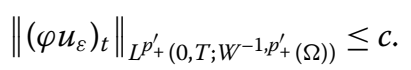


For a fixed $s$ such that $s>\frac{N}{2}+1$, one has $H_{0}^{s}(\Omega) \hookrightarrow W^{1, p_{+}}(\Omega)$. Consequently, $W^{-1, p_{+}^{\prime}}(\Omega) \hookrightarrow H^{-s}(\Omega)$. As a result, we have

$$
\left\|\left(\varphi u_{\varepsilon}\right)_{t}\right\|_{L^{p_{+}^{\prime}\left(0, T ; H^{-S}(\Omega)\right)}} \leq c
$$

At the same time, we have

$$
\iint_{Q_{T}}\left|\nabla\left(\varphi u_{\varepsilon}\right)\right|^{p_{-}} d x d t \leq c(\varphi)\left(1+\int_{0}^{T} \int_{\Omega_{\varphi}}\left|\nabla u_{\varepsilon}\right|^{p_{-}} d x d t\right) \leq c(\varphi)
$$

where $\Omega_{\varphi}=\operatorname{supp} \varphi$. We give an explanation of this inequality. Since $a(x) \in C^{1}(\bar{\Omega}), a(x)>0$ when $x \in \Omega$, for any $\varphi(x) \in C_{0}^{1}(\Omega)$,

$$
\frac{\varphi(x)}{a(x)} \leq c(\varphi)
$$

then

$$
\begin{aligned}
& \int_{0}^{T} \int_{\Omega_{\varphi}}\left|\nabla u_{\varepsilon}\right|^{p_{-}} d x d t \\
& \quad=\int_{0}^{T} \int_{\Omega} \varphi(x)\left|\nabla u_{\varepsilon}\right|^{p_{-}} d x d t \\
& \quad=\int_{0}^{T} \int_{\Omega} \frac{\varphi(x)}{a(x)} a(x)\left|\nabla u_{\varepsilon}\right|^{p_{-}} d x d t \\
& \quad \leq c(\varphi) \int_{0}^{T} \int_{\Omega} a(x)\left|\nabla u_{\varepsilon}\right|^{p_{-}} d x d t \\
& \leq c(\varphi) .
\end{aligned}
$$

Thus we have

$$
\left\|\varphi u_{\varepsilon}\right\|_{L^{p_{+}^{\prime}}\left(0, T ; W_{0}^{1, p_{-}}(\Omega)\right)} \leq c
$$

Noticing that $W_{0}^{1, p_{-}}(\Omega) \hookrightarrow L^{p_{-}}(\Omega) \hookrightarrow H^{-s}(\Omega)$, we can employ Aubin's compactness theorem in [12] to obtain $\varphi u_{\varepsilon} \rightarrow \varphi u$ strongly in $L^{p_{+}^{\prime}}\left(0, T ; L^{p_{-}}(\Omega)\right)$. Thus $\varphi u_{\varepsilon} \rightarrow \varphi u$ a.e. in $Q_{T}$. In particular, due to the arbitrariness of $\varphi, u_{\varepsilon} \rightarrow u$ a.e. in $Q_{T}$.

Now, by (2.7),

$$
\varepsilon\left|\nabla u_{\varepsilon}\right|^{p_{+}-2} \nabla u_{\varepsilon} \rightarrow 0, \quad \text { in } L^{\frac{p_{+}}{p_{+}-1}}\left(Q_{T}\right)
$$

By (2.4), (2.5), (2.6), there exists a function $u$ and an $n$-dimensional vector function $\vec{\zeta}=$ $\left(\zeta_{1}, \ldots, \zeta_{n}\right)$ satisfying

$$
u \in L^{\infty}\left(Q_{T}\right), \quad\left|\zeta_{i}\right| \in L^{\frac{p(x)}{p(x)-1}}\left(Q_{T}\right)
$$

and

$$
u_{\varepsilon} \rightarrow u, \quad \text { weakly-star in } L^{\infty}\left(Q_{T}\right), \quad u_{\varepsilon} \rightarrow u, \quad \text { a.e. in } Q_{T} \text {, }
$$




$$
\begin{aligned}
& u_{\varepsilon}^{q} \rightarrow u^{q}, \quad \text { a.e. in } Q_{T}, \\
& u_{\varepsilon x_{i}} \rightarrow u_{x_{i}}, \quad \text { in } L_{\mathrm{loc}}^{p(x)}\left(Q_{T}\right), \\
& a(x)\left|\nabla u_{\varepsilon}\right|^{p(x)-2} \nabla u_{\varepsilon} \rightarrow \vec{\zeta}, \quad \text { in }\left\{L^{1}\left(0, T ; L^{\frac{p(x)}{p(x)-1}}(\Omega)\right)\right\}^{N} .
\end{aligned}
$$

Moreover, we can prove that

$$
\iint_{Q_{T}} a|\nabla u|^{p(x)-2} \nabla u \cdot \nabla \varphi d x d t=\iint_{Q_{T}} \vec{\zeta} \cdot \nabla \varphi d x d t
$$

for any given function $\varphi \in C_{0}^{1}\left(Q_{T}\right)$.

Then, by (2.14), for any $\varphi \in C_{0}^{1}\left(Q_{T}\right)$, we have

$$
\left\langle u_{t}, \varphi\right\rangle+\iint_{Q_{T}}\left[a(x)|\nabla u|^{p(x)-2} \nabla u \nabla \varphi+u^{q}\left(b^{i}(x) \varphi_{x_{i}}+b_{x_{i}}^{i}(x) \varphi\right)\right] d x d t=0 .
$$

If for any given $t \in[0, T)$, denoting $\Omega_{\varphi}=\operatorname{supp} \varphi$, then we have

$$
\left\langle u_{t}, \varphi\right\rangle+\int_{0}^{T} \int_{\Omega_{\varphi}}\left[a(x)|\nabla u|^{p(x)-2} \nabla u \nabla \varphi+u^{q}\left(b^{i}(x) \varphi_{x_{i}}+b_{x_{i}}^{i}(x) \varphi\right)\right] d x d t=0 .
$$

Moreover, for any $\varphi_{1} \in C_{0}^{1}\left(Q_{T}\right), \varphi_{2}(x, t) \in L^{\infty}\left(0, T ; W_{\text {loc }}^{1, p(x)}(\Omega)\right)$, we clearly have

$$
g\left(\varphi_{1} \varphi_{2}\right) \in L^{\infty}\left(0, T ; W_{0}^{1, p(x)}\left(\Omega_{\varphi_{1}}\right)\right)
$$

Since $C_{0}^{\infty}\left(\Omega_{\varphi_{1}}\right)$ is dense in $W_{0}^{1, p(x)}\left(\Omega_{\varphi_{1}}\right)$, by taking a limit, we have

$$
\begin{aligned}
& \left\langle u_{t}, g\left(\varphi_{1} \varphi_{2}\right)\right\rangle+\int_{0}^{T} \int_{\Omega_{\varphi_{1}}}\left[a(x)|\nabla u|^{p(x)-2} \nabla u \nabla g\left(\varphi_{1} \varphi_{2}\right)\right] d x d t \\
& \quad+\int_{0}^{T} \int_{\Omega_{\varphi_{1}}}\left[u^{q}\left(b^{i}(x) g_{x_{i}}\left(\varphi_{1} \varphi_{2}\right)+b_{x_{i}}^{i} g\left(\varphi_{1} \varphi_{2}\right)\right)\right] d x d t \\
& =0
\end{aligned}
$$

and so

$$
\begin{aligned}
& \left\langle u_{t}, g\left(\varphi_{1} \varphi_{2}\right)\right\rangle+\int_{0}^{T} \int_{\Omega}\left[a(x)|\nabla u|^{p(x)-2} \nabla u \nabla g\left(\varphi_{1} \varphi_{2}\right)\right] d x d t \\
& \quad+\int_{0}^{T} \int_{\Omega}\left[u^{q}\left(b^{i}(x) g_{x_{i}}\left(\varphi_{1} \varphi_{2}\right)+b_{x_{i}}^{i} g\left(\varphi_{1} \varphi_{2}\right)\right)\right] d x d t \\
& =0 .
\end{aligned}
$$

Finally, the initial value condition in the sense of (1.7) can be proved (1.7) as in [2], then $u$ is a solution of Eq. (1.1) with the initial value (1.2) in the sense of Definition 1.3. Thus we have Theorem 1.4 . 


\section{The proof of Theorem 1.5}

Lemma 3.1 Let $v \in L^{p_{+}}\left(0, T ; W_{0}^{1, p_{+}}(\Omega)\right)$, $v_{t} \in L^{p_{+}^{\prime}}\left(0, T ; W^{-1, p_{+}{ }^{\prime}}(\Omega)\right)$. For any continuous function $h(s), H(s)=\int_{0}^{s} h(s) d s$, a.e. $t_{1}, t_{2} \in[0, T)$,

$$
\int_{t_{1}}^{t_{2}}\left\langle v_{t}, h(v)\right\rangle d t=\int_{\Omega}\left(H(v)\left(x, t_{2}\right)-H(v)\left(x, t_{1}\right)\right) d x .
$$

Lemma 3.1 is proved in the note [2].

It is well known that the $B V$ function is defined as $u \in B V(\Omega)=\left\{u \in L^{1}(\Omega): \int_{\Omega}|\nabla u| d x<\right.$ $\infty\}=W^{1,1}(\Omega)$. Moreover, $C_{0}^{\infty}(\Omega)$ is dense in the space $W_{0}^{1,1}(\Omega)=\left\{u: u \in B V(\Omega),\left.u\right|_{\partial \Omega}=0\right\}$, accordingly, $L^{p^{0}}\left(0, T ; W_{0}^{1, p^{0}}(\Omega)\right)$ is dense in $L^{p^{0}}\left(0, T ; W_{0}^{1,1}(\Omega)\right)$. Then we have the following.

Remark 3.2 Lemma 3.1 is still true for the function $v \in L^{p_{+}}\left(0, T ; W_{0}^{1,1}(\Omega)\right), v_{t} \in L^{p_{+}^{\prime}}(0, T$; $\left.W^{-1, p_{+}{ }^{\prime}}(\Omega)\right)$.

Now, we begin to prove Theorem 1.4. For small $\eta>0$, let

$$
S_{\eta}(s)=\int_{0}^{s} h_{\eta}(\tau) d \tau, \quad h_{\eta}(s)=\frac{2}{\eta}\left(1-\frac{|s|}{\eta}\right)_{+} .
$$

Obviously, $h_{\eta}(s) \in C(\mathbb{R}), S_{\eta}(s)$ is monotone increasing about $\eta$ and

$$
\lim _{\eta \rightarrow 0} S_{\eta}(s)=\operatorname{sgn} s, \quad \lim _{\eta \rightarrow 0} H_{\eta}(s)=\int_{0}^{s} S_{\eta}(\tau) d \tau=|s|,
$$

moreover, one has

$$
h_{\eta}(s) \geq 0, \quad\left|s h_{\eta}(s)\right| \leq 1, \quad \lim _{\eta \rightarrow 0} s S_{\eta}^{\prime}(s)=0 .
$$

For two solutions $u(x, t), v(x, t)$ of Eq. (1.1) with the initial values

$$
u(x, 0)=u_{0}(x), \quad v(x, 0)=v_{0}(x)
$$

respectively, we can choose $S_{\eta}\left(a^{\beta}(u-v)\right)$ as the test function, where $\beta \geq 1$ is a constant. Then

$$
\begin{aligned}
& \int_{0}^{t} \int_{\Omega} S_{\eta}\left(a^{\beta}(u-v)\right) \frac{\partial(u-v)}{\partial t} d x d t \\
& \quad+\int_{0}^{t} \int_{\Omega} a^{\beta+1}(x)\left(|\nabla u|^{p(x)-2} \nabla u-|\nabla v|^{p(x)-2} \nabla v\right) \cdot \nabla(u-v) S_{\eta}^{\prime}\left(a^{\beta}(u-v)\right) d x d t \\
& \quad+\int_{0}^{t} \int_{\Omega} a(x)\left(|\nabla u|^{p(x)-2} \nabla u-|\nabla v|^{p(x)-2} \nabla v\right) \cdot \nabla a^{\beta}(u-v) S_{\eta}^{\prime}\left(a^{\beta}(u-v)\right) d x d t \\
& \quad+\int_{0}^{t} \int_{\Omega}\left(b_{x_{i}}^{i}(x)\right)\left(u^{q}-v^{q}\right) S_{\eta}\left(a^{\beta}(u-v)\right)+a^{\beta} b^{i}(x)\left(u^{q}-v^{q}\right) \\
& \quad+(u-v)_{x_{i}} S_{\eta}^{\prime}\left(a^{\beta}(u-v)\right) d x d t \\
& \quad+\int_{0}^{t} \int_{\Omega} b^{i}(x)\left(u^{q}-v^{q}\right) \cdot a_{x_{i}}^{\beta}(u-v) S_{\eta}^{\prime}\left(a^{\beta}(u-v)\right) d x d t \\
& =0 .
\end{aligned}
$$


Let us analyze every term on the left hand side of (3.4). In the first place, since $a(x) \geq 0$, $S_{\eta}^{\prime}(s) \geq 0$, for the second term, one has

$$
\int_{\Omega} a^{\beta+1}(x)\left(|\nabla u|^{p(x)-2} \nabla u-|\nabla v|^{p(x)-2} \nabla v\right) \cdot \nabla(u-v) S_{\eta}^{\prime}\left(a^{\beta}(u-v)\right) d x \geq 0 .
$$

For the third term, since $|\nabla a(x)| \leq c$ in $\Omega$, we have

$$
\begin{aligned}
& \left|\int_{\Omega} a^{\beta}(u-v) S_{\eta}^{\prime}\left(a^{\beta}(u-v)\right)\left(|\nabla u|^{p(x)-2} \nabla u-|\nabla v|^{p(x)-2} \nabla v\right) d x\right| \\
& =\mid \int_{\left\{\Omega: a^{\beta}|u-v|<\eta\right\}} a^{-\frac{p(x)-1}{p(x)}} a^{\beta}(u-v) S_{\eta}^{\prime}\left(a^{\beta}(u-v)\right) \\
& \quad \cdot a^{\frac{p(x)-1}{p(x)}}\left(|\nabla u|^{p(x)-2} \nabla u-|\nabla v|^{p(x)-2} \nabla v\right) d x \mid \\
& \leq\left(\int_{\left\{\Omega: a^{\beta}|u-v|<\eta\right\}}\left|a^{-\frac{p(x)-1}{p(x)}} a^{\beta}(u-v) S_{\eta}^{\prime}\left(a^{\beta}(u-v)\right)\right|^{p(x)} d x\right)^{\frac{1}{p_{1}}} \\
& \quad \cdot\left(\int_{\left\{\Omega: a^{\beta}|u-v|<\eta\right\}} a(x)\left(|\nabla u|^{p(x)}+|\nabla v|^{p(x)}\right) d x\right)^{\frac{1}{p_{1}^{\prime}}},
\end{aligned}
$$

where $p_{1}$ is $p_{+}$or $p_{-}$according to Lemma $1.2, p^{\prime}(x)=\frac{p(x)}{p(x)-1}, p_{1}^{\prime}=p_{+}^{\prime}$ or $p_{-}^{\prime}$ has a similar meaning.

If $\{x \in \Omega: u-v=0\}$ has zero measure, then

$$
\lim _{\eta \rightarrow 0} \int_{\left\{\Omega: a^{\beta}|u-v|<\eta\right\}} a(x)\left(|\nabla u|^{p(x)}+|\nabla v|^{p(x)}\right) d x=0 .
$$

Since (1.11),

$$
\int_{\Omega} a^{-(p(x)-1)}(x) d x<\infty
$$

by the fact $\left|a^{\beta}(u-v) S_{\eta}^{\prime}\left(a^{\beta}(u-v)\right)\right| \leq c$, it implies

$$
\left.\left|\int_{\left\{\Omega: a^{\beta}|u-v|<\eta\right\}}\right| a^{-\frac{p(x)-1}{p(x)}} a^{\beta}(u-v) S_{\eta}^{\prime}\left(a^{\beta}(u-v)\right)\right|^{p(x)} d x \mid \leq c,
$$

by (3.6), one has

$$
\lim _{\eta \rightarrow 0}\left|\int_{\Omega} a^{\beta}(u-v) S_{\eta}^{\prime}\left(a^{\beta}(u-v)\right)\left(|\nabla u|^{p(x)-2} \nabla u-|\nabla v|^{p(x)-2} \nabla v\right) d x\right|=0 .
$$

If $\{x \in \Omega: u-v=0\}$ has a positive measure, since

$$
\begin{gathered}
\int_{\left\{\Omega: a^{\beta}|u-v|<\eta\right\}}\left|a^{-\frac{p(x)-1}{p(x)}} a^{\beta}(u-v) S_{\eta}^{\prime}\left(a^{\beta}(u-v)\right)\right|^{p(x)} d x \\
\leq \int_{\Omega}\left|a^{-\frac{p(x)-1}{p(x)}} a^{\beta}(u-v) S_{\eta}^{\prime}\left(a^{\beta}(u-v)\right)\right|^{p(x)} d x,
\end{gathered}
$$


and

$$
\int_{\left\{\Omega: a^{\beta}|u-v|<\eta\right\}} a(x)\left(|\nabla u|^{p(x)}+|\nabla v|^{p(x)}\right) d x \leq \int_{\Omega} a(x)\left(|\nabla u|^{p(x)}+|\nabla v|^{p(x)}\right) d x \leq c,
$$

according to the condition (1.11) and the fact $\left|a^{\beta}(u-v) S_{\eta}^{\prime}\left(a^{\beta}(u-v)\right)\right| \leq c$, using the dominated convergence theorem and Hölder inequality, by the last formula of (3.3), one has

$$
\lim _{\eta \rightarrow 0}\left(\int_{\left\{\Omega: a^{\beta}|u-v|<\eta\right\}}\left|a^{-\frac{p(x)-1}{p(x)}} a^{\beta}(u-v) S_{\eta}^{\prime}\left(a^{\beta}(u-v)\right)\right|^{p(x)} d x\right)^{\frac{1}{p_{1}}}=0 .
$$

Thus, from (3.6), (3.9) is true.

Moreover, since $u, v \in L^{\infty}\left(Q_{T}\right)$, by (1.10), using the dominated convergence theorem, we have

$$
\begin{aligned}
& \lim _{\eta \rightarrow 0}\left|\int_{\Omega} b^{i}(x)\left(u^{q}-v^{q}\right) \cdot a_{x_{i}}^{\beta}(u-v) S_{\eta}^{\prime}\left(a^{\beta}(u-v)\right) d x\right| \\
& \quad \leq \lim _{\eta \rightarrow 0} \int_{\Omega}\left|b^{i}(x)\left(u^{q}-v^{q}\right)\right| S_{\eta}^{\prime}\left(a^{\beta}(u-v)\right)\left|a^{\beta}(u-v) \frac{a_{x_{i}}^{\beta}}{a^{\beta}}\right| d x \\
& \quad \leq c \lim _{\eta \rightarrow 0} \int_{\Omega}\left|S_{\eta}^{\prime}\left(a^{\beta}(u-v)\right) a^{\beta}(u-v)\right| \frac{\left|b^{i}(x) \nabla a\right|}{a} d x \\
& \quad \leq c \lim _{\eta \rightarrow 0}\left(\int_{\Omega}\left|S_{\eta}^{\prime}\left(a^{\beta}(u-v)\right) a^{\beta}(u-v)\right| d x\right)^{\frac{1}{2}}\left(\int_{\Omega}\left|\frac{b^{i}(x) \nabla a}{a}\right|^{2} d x\right)^{\frac{1}{2}} \\
& \quad=0 .
\end{aligned}
$$

Since $q \geq 1$,

$$
\left|u^{q}-v^{q}\right| \leq c|u-v|
$$

and by (1.10), $\left|a^{-\frac{1}{p}} b^{i}(x)\right| \leq c$,

$$
\begin{aligned}
& \left|\int_{\Omega} b^{i}(x) a^{\beta}(x)\left(u^{q}-v^{q}\right) S_{\eta}{ }^{\prime}\left(a^{\beta}(u-v)\right)(u-v)_{x_{i}} d x\right| \\
& \leq c\left(\int_{\Omega} a(x)\left(|\nabla u|^{p(x)}+|\nabla v|^{p(x)}\right) d x\right)^{\frac{1}{p_{1}}} \\
& \quad \cdot\left(\int_{\Omega}\left|a^{-\frac{1}{p(x)}} b^{i}(x)\right|^{\frac{p(x)}{p(x)-1}}\left|a^{\beta}(x)(u-v) S_{\eta}{ }^{\prime}\left(a^{\beta}(u-v)\right)\right|^{\frac{p(x)}{p(x)-1}} d x\right)^{\frac{1}{p_{1}^{\prime}}} \\
& \leq c\left(\int_{\Omega}\left|a^{-\frac{1}{p}} b^{i}(x)\right|^{\frac{p(x)}{p(x)-1}}\left|a^{\beta}(x)(u-v) S_{\eta}{ }^{\prime}\left(a^{\beta}(u-v)\right)\right|^{\frac{p(x)}{p(x)-1}} d x\right)^{\frac{1}{p_{1}^{\prime}}} .
\end{aligned}
$$

Therefore,

$$
\lim _{\eta \rightarrow 0}\left|\int_{\Omega} b^{i}(x)\left(u^{q}-v^{q}\right) S_{\eta}{ }^{\prime}\left(a^{\beta}(u-v)\right)(u-v)_{x_{i}} a^{\beta}(x) d x\right|=0
$$


At the same time,

$$
\lim _{\eta \rightarrow 0}\left|\int_{\Omega} b_{x_{i}}^{i}(x)\left(u^{q}-v^{q}\right) S_{\eta}\left(a^{\beta}(u-v)\right) d x\right| \leq c \int_{\Omega}|u-v| d x,
$$

obviously by the assumption of $b^{i}(x) \in C^{1}(\bar{\Omega})$.

Finally, for the first term on the left hand side of (3.4), by the monotone convergent theorem, one has

$$
\begin{gathered}
\lim _{\eta \rightarrow 0} \int_{0}^{t} \int_{\Omega} S_{\eta}\left(a^{\beta}(u-v)\right) \frac{\partial(u-v)}{\partial t} d x d t \\
=\int_{0}^{t} \int_{\Omega} \operatorname{sign}\left(a^{\beta}(u-v)\right) \frac{\partial(u-v)}{\partial t} d x d t \\
=\lim _{\eta \rightarrow 0} \int_{0}^{t} \int_{\Omega} S_{\eta}(u-v) \frac{\partial(u-v)}{\partial t} d x d t .
\end{gathered}
$$

We can employ Remark 3.2 to deduce that

$$
\begin{aligned}
\lim _{\eta \rightarrow 0} \int_{0}^{t} \int_{\Omega} S_{\eta}(u-v) \frac{\partial(u-v)}{\partial t} d x d t \\
=\lim _{\eta \rightarrow 0} \int_{0}^{t}\left(H_{\eta}(u-v)(t)-H_{\eta}\left(u_{0}-v_{0}\right)\right) d t \\
=\int_{\Omega}|u(x, t)-v(x, t)| d x-\int_{\Omega}\left|u_{0}(x)-v_{0}(x)\right| d x .
\end{aligned}
$$

Now, let $\eta \rightarrow 0$ in (3.4). By (3.5), (3.9), (3.11), (3.12), (3.13) and (3.14), one has

$$
\int_{\Omega}|u(x, t)-v(x, t)| d x \leq c \int_{\Omega}\left|u_{0}(x)-v_{0}(x)\right| d x, \quad \forall t \in[0, T) .
$$

\section{The proofs of Theorem 1.6 and Theorem 1.8}

Proof of Theorem 1.6 For a small positive constant $\lambda>0$, denoting $\Omega_{\lambda}=\{x \in \Omega: a(x)>\lambda\}$ as before, let

$$
\phi_{\lambda}(x)= \begin{cases}1, & \text { if } x \in \Omega_{\lambda} \\ \frac{1}{\lambda} a(x), & \text { if } x \in \Omega \backslash \Omega_{\lambda}\end{cases}
$$

Now, by a process of limit, for $g(s)=s$, we can choose $\varphi_{1}=\phi_{\lambda}(x) \chi_{[0, t]}, \varphi_{2}=S_{\eta}(u-v)$, and integrate it over $Q_{T}$, accordingly,

$$
\begin{aligned}
& \int_{0}^{t} \int_{\Omega} \phi_{\lambda}(x) S_{\eta}(u-v) \frac{\partial(u-v)}{\partial t} d x d t \\
& \quad+\int_{0}^{t} \int_{\Omega} \phi_{\lambda}(x) a(x)\left(|\nabla u|^{p(x)-2} \nabla u-|\nabla v|^{p(x)-2} \nabla v\right) \\
& \quad \cdot \nabla(u-v) S_{\eta}^{\prime}(u-v) d x d t \\
& \quad+\int_{0}^{t} \int_{\Omega} a(x)\left(|\nabla u|^{p(x)-2} \nabla u-|\nabla v|^{p(x)-2} \nabla v\right) \cdot \nabla \phi_{\lambda}(x) S_{\eta}(u-v) d x d t
\end{aligned}
$$


Chan Boundary Value Problems ( 2018) 2018:104

Page 12 of 15

$$
\begin{aligned}
& +\int_{0}^{t} \int_{\Omega}\left(u^{q}-v^{q}\right)\left[b^{i}(x)\left(\phi_{\lambda}(x) S_{\eta}(u-v)\right)_{x_{i}}\right] d x d t \\
& +\int_{0}^{t} \int_{\Omega}\left(u^{q}-v^{q}\right)\left[b_{x_{i}}^{i}(x) \phi_{\lambda}(x) S_{\eta}(u-v)\right] d x d t=0 .
\end{aligned}
$$

In the first place,

$$
\int_{\Omega} \phi_{\lambda}(x) a(x)\left(|\nabla u|^{p(x)-2} \nabla u-|\nabla v|^{p(x)-2} \nabla v\right) \cdot \nabla(u-v) S_{\eta}^{\prime}(u-v) d x \geq 0
$$

is clear. For the third term,

$$
\begin{aligned}
& \left|\int_{\Omega} a(x)\left(|\nabla u|^{p(x)-2} \nabla u-|\nabla v|^{p(x)-2} \nabla v\right) \cdot \nabla \phi_{\lambda}(x) S_{\eta}(u-v) d x\right| \\
& \quad \leq \int_{\Omega \backslash \Omega_{\lambda}} a(x)\left|\left(|\nabla u|^{p(x)-2} \nabla u-|\nabla v|^{p(x)-2} \nabla v\right) \cdot \nabla \phi_{\lambda}(x) S_{\eta}(u-v)\right| d x \\
& \quad \leq \int_{\Omega \backslash \Omega_{\lambda}} a(x)\left|\left(|\nabla u|^{p(x)-2} \nabla u-|\nabla v|^{p(x)-2} \nabla v\right)\right|\left|\nabla \phi_{\lambda}(x)\right| d x \\
& \quad \leq \frac{c}{\lambda}\left[\int_{\Omega \backslash \Omega_{\lambda}} a(x)|\nabla u|^{p(x)-1}|\nabla a| d x+\int_{\Omega \backslash \Omega_{\lambda}} a(x)|\nabla v|^{p(x)-1}|\nabla a| d x\right] .
\end{aligned}
$$

By (1.14), i.e.

$$
\frac{c}{\lambda}\left(\lambda \int_{\Omega \backslash \Omega_{\lambda}}|\nabla a|^{p(x)} d x\right)^{\frac{1}{p_{+}}} \leq c,
$$

using the Hölder inequality, by (4.4),

$$
\begin{aligned}
& \left|\int_{\Omega} a(x)\left(|\nabla u|^{p(x)-2} \nabla u-|\nabla v|^{p(x)-2} \nabla v\right) \cdot \nabla \phi_{\lambda}(x) S_{\eta}(u-v) d x\right| \\
& \leq \frac{c}{\lambda}\left[\int_{\Omega \backslash \Omega_{\lambda}} a(x)|\nabla u|^{p(x)-1}|\nabla a| d x+\int_{\Omega \backslash \Omega_{\lambda}} a(x)|\nabla v|^{p(x)-1}|\nabla a| d x\right] \\
& \leq \frac{c}{\lambda}\left(\int_{\Omega \backslash \Omega_{\lambda}} a|\nabla a|^{p(x)} d x\right)^{\frac{1}{p_{+}}}\left(\int_{\Omega \backslash \Omega_{\lambda}} a(x)|\nabla u|^{p(x)} d x\right)^{\frac{1}{p_{+}^{\prime}}} \\
& \quad+\frac{c}{\lambda}\left(\int_{\Omega \backslash \Omega_{\lambda}} a(x)|\nabla a|^{p(x)} d x\right)^{\frac{1}{p_{+}}}\left(\int_{\Omega \backslash \Omega_{\lambda}} a(x)|\nabla v|^{p(x)} d x\right)^{\frac{1}{p_{+}^{\prime}}} \\
& \leq c\left(\int_{\Omega \backslash \Omega_{\lambda}} a(x)|\nabla u|^{p(x)} d x\right)^{\frac{1}{p_{+}^{\prime}}}+c\left(\int_{\Omega \backslash \Omega_{\lambda}} a(x)|\nabla v|^{p(x)} d x\right)^{\frac{1}{p_{+}}} .
\end{aligned}
$$

By (4.5), clearly,

$$
\lim _{\lambda \rightarrow 0}\left|\int_{\Omega} a(x)\left(|\nabla u|^{p(x)-2} \nabla u-|\nabla v|^{p(x)-2} \nabla v\right) \cdot \nabla \phi_{\lambda}(x) S_{\eta}(u-v) d x\right|=0 .
$$

As for the fourth term, by $q \geq 1$, and the condition (1.13)

$$
\int_{\Omega} a^{-\frac{1}{p(x)-1}}(x) d x<c
$$


using the last formula of (3.3) and the dominated convergence theorem, one has

$$
\begin{aligned}
& \lim _{\eta \rightarrow 0}\left|\int_{\Omega} \phi_{\lambda} b^{i}(x)\left(u^{q}-v^{q}\right) S_{\eta}^{\prime}(u-v)(u-v)_{x_{i}} d x\right| \\
& \quad \leq c \lim _{\eta \rightarrow 0}\left(\int_{\Omega}\left|a^{-\frac{1}{p(x)}} S_{\eta}^{\prime}(u-v)(u-v)\right|^{\frac{p(x)}{p(x)-1}} d x\right)^{\frac{1}{p_{1}^{\prime}}}\left(\int_{\Omega} a(x)\left(|\nabla u|^{p(x)}+|\nabla v|^{p(x)}\right) d x\right)^{\frac{1}{p_{1}}} \\
& \quad=0
\end{aligned}
$$

where $p_{1}=p_{+}$or $p_{-}$according to (ii) of Lemma 1.2, $p_{1}^{\prime}$ has a similar meaning. We have

$$
\begin{aligned}
& \lim _{\lambda \rightarrow 0}\left|\int_{\Omega} \phi_{\lambda x_{i}} b^{i}(x)\left(u^{q}-v^{q}\right) S_{\eta}(u-v) d x\right| \\
& \quad \leq \lim _{\lambda \rightarrow 0} \frac{c}{\lambda} \int_{\Omega \backslash \Omega_{\lambda}}|\nabla a| d x \\
& \quad \leq \lim _{\lambda \rightarrow 0} \frac{c}{\lambda}\left(\int_{\Omega \backslash \Omega_{\lambda}} a(x)|\nabla a|^{p(x)} d x\right)^{\frac{1}{p_{1}}}\left(\int_{\left.\Omega_{\backslash \Omega_{\lambda}} a^{-\frac{1}{p(x)-1}}(x) d x\right)^{\frac{1}{p_{+}^{\prime}}}}\right. \\
& \quad=0 .
\end{aligned}
$$

By (4.7)-(4.8), for the fourth term on the left term in (4.2), one has

$$
\lim _{\eta \rightarrow 0}\left|\int_{\Omega}\left(u^{q}-v^{q}\right)\left[b^{i}(x)\left(\phi_{\lambda}(x) S_{\eta}(u-v)\right)_{x_{i}}\right] d x\right| \leq c \int_{\Omega}|u-v| d x .
$$

Moreover, for the fifth term, one has

$$
\lim _{\eta \rightarrow 0}\left|\int_{\Omega}\left(u^{q}-v^{q}\right) b_{x_{i}}^{i}(x) \phi_{\lambda}(x) S_{\eta}(u-v) d x\right| \leq c \int_{\Omega}|u-v| d x .
$$

Finally, for the first term, also by the monotone convergent theorem, and employing Remark 3.2, one has

$$
\begin{aligned}
& \lim _{\eta \rightarrow 0} \lim _{\lambda \rightarrow 0} \int_{0}^{t} \int_{\Omega} \phi_{\lambda}(x) S_{\eta}(u-v) \frac{\partial(u-v)}{\partial t} d x d t \\
& \quad=\int_{\Omega}|u(x, t)-v(x, t)| d x-\int_{\Omega}\left|u_{0}(x)-v_{0}(x)\right| d x .
\end{aligned}
$$

Now, after letting $\lambda \rightarrow 0$, let $\eta \rightarrow 0$ in (4.2). By (4.3), (4.6), (4.9), (4.10) and (4.11), using the Gronwall inequality, we have

$$
\int_{\Omega}|u(x, t)-v(x, t)| d x \leq c \int_{\Omega}\left|u_{0}(x)-v_{0}(x)\right| d x .
$$

Proof of Theorem 1.8 Similar to the proof of Theorem 1.5, we have (4.2) and (4.3). Since $u(x)$ and $v(x)$ satisfy the condition(1.15), by (4.4), using the Hölder inequality,

$$
\begin{aligned}
& \left|\int_{\Omega} a(x)\left(|\nabla u|^{p(x)-2} \nabla u-|\nabla v|^{p(x)-2} \nabla v\right) \cdot \nabla \phi_{\lambda}(x) S_{\eta}(u-v) d x\right| \\
& \quad \leq \frac{c}{\lambda}\left(\int_{\Omega \backslash \Omega_{\lambda}} a|\nabla a|^{p(x)} d x\right)^{\frac{1}{p_{+}}}\left(\int_{\Omega_{\backslash \Omega}} a(x)|\nabla u|^{p(x)} d x\right)^{\frac{1}{p_{+}^{\prime}}}
\end{aligned}
$$




$$
\begin{aligned}
& +\frac{c}{\lambda}\left(\int_{\Omega \backslash \Omega_{\lambda}} a(x)|\nabla a|^{p(x)} d x\right)^{\frac{1}{p_{+}}}\left(\int_{\Omega \backslash \Omega_{\lambda}} a(x)|\nabla v|^{p(x)} d x\right)^{\frac{1}{p_{+}}} \\
\leq & c\left(\int_{\Omega \backslash \Omega_{\lambda}} a|\nabla a|^{p(x)} d x\right)^{\frac{1}{p_{+}}}+c\left(\int_{\Omega \backslash \Omega_{\lambda}} a(x)|\nabla a|^{p(x)} d x\right)^{\frac{1}{p_{+}}},
\end{aligned}
$$

which goes to zero as $\lambda \rightarrow 0$ since $a(x) \in C^{1}(\bar{\Omega})$ satisfying (1.15), one has (4.6). Last but not least, since $\int_{\Omega} a^{-\frac{1}{p(x)-1}}(x) d x<\infty$, similar to the proof of Theorem 1.5, one has (4.7)-(4.11), and we know that the stability (1.12) is true. Theorem 1.8 is proved.

\section{Conclusion}

The equations addressed arise in electrorheological fluids theory. Compared with the known results $[2,3,14]$, the novelty lies in that, if the diffusion coefficient is degenerate on the boundary, the existence and the uniqueness of the weak solutions may be proved independent of the boundary value condition. Moreover, how the convection term with an oriented form affects the well-posedness of the solutions of the equation is studied.

Acknowledgements

The author would like to thank BVOP for kindly considering my paper to be published.

\section{Funding}

The paper is supported by the Natural Science Foundation of Fujian province and supported by the Science Foundation of Xiamen University of Technology, China.

\section{Abbreviations}

Not applicable.

\section{Availability of data and materials}

Not applicable.

Ethics approval and consent to participate

Not applicable.

\section{Competing interests}

The author declares that he has no competing interests.

\section{Authors' contributions}

The author read and approved the final manuscript.

\section{Publisher's Note}

Springer Nature remains neutral with regard to jurisdictional claims in published maps and institutional affiliations.

Received: 8 May 2018 Accepted: 2 July 2018 Published online: 05 July 2018

\section{References}

1. Ruzicka, M.: Electrorheological Fluids: Modeling and Mathematical Theory. Lecture Notes in Math., vol. 1748. Springer, Berlin (2000)

2. Antontsev, S., Shmarev, S.: Anisotropic parabolic equations with variable nonlinearity. Publ. Mat. 53, 355-399 (2009)

3. Gala, S., Ragusa, M.A.: On the regularity criterion of weak solutions for the 3D MHD equations. Z. Angew. Math. Phys. 68(6), Article ID 140 (2017)

4. Gao, W., Guo, B.: Existence and localization of weak solutions of nonlinear parabolic equations with variable exponent of nonlinearity. Ann. Mat. Pura Appl. 191, 551-562 (2012)

5. Guo, B., Gao, W.: Study of weak solutions for parabolic equations with nonstandard growth conditions. J. Math. Anal. Appl. 374(2), 374-384 (2011)

6. Bennouna, J., El Hamdaoui, B., Mekkour, M., Redwane, H.: Nonlinear parabolic inequalities in Lebesgue-Sobolev spaces with variable exponent. Ric. Mat. 65(1), 93-125 (2016)

7. Bendahmane, M., Wittbold, P., Zimmermann, A.: Renormalized solutions for a nonlinear parabolic equation with variable exponents and $L^{1}$ data. J. Differ. Equ. 249(6), 1483-1515 (2010)

8. Wu, Z., Zhao, J., Yun, J., Li, F.: Nonlinear Diffusion Equations. World Scientific, Singapore (2001)

9. Zhan, H., Wen, J.: Well-posedness of weak solutions to electrorheological fluid equations with degeneracy on the boundary. Electron. J. Differ. Equ. 2017, 13 (2017) 
10. Zhan, H.: The boundary value condition of an evolutionary $p(x)$-Laplacian equation. Bound. Value Probl. 2015, 112 (2015). https://doi.org/10.1186/s13661-015-0377-6

11. Zhan, H., Xu, B.: A new kind of weak solution of non-Newtonian fluid equation. J. Funct. Spaces 2017, Article ID 7916730 (2017). https://doi.org/10.1155/2017/7916730

12. Zhikov, V.V.: On the density of smooth functions in Sobolev-Orlicz spaces. Otdel. Mat. Inst. Steklov. (POMI) 310, 67-81 (2004). Translation in J. Math. Sci. (N.S.) 132, 285-294 (2006)

13. Yin, J., Wang, C.: Properties of the boundary flux of a singular diffusion process. Chin. Ann. Math. 25B(2), 175-182 (2004)

14. Alghamdi, A.M., Gala, S., Ragusa, M.A.: A regularity criterion of weak solutions to the 3D Boussinesq equations. AIMS Math. 2(3), 451-457 (2017)

Submit your manuscript to a SpringerOpen ${ }^{\circ}$ journal and benefit from:

- Convenient online submission

- Rigorous peer review

- Open access: articles freely available online

- High visibility within the field

- Retaining the copyright to your article

Submit your next manuscript at $\gg$ springeropen.com 